Bull. Austral. Math. Soc.

VOL. 48 (1993) [485-494]

\title{
LANDAU-KOLMOGOROV INEQUALITY ON A FINITE INTERVAL
}

\author{
W. CHEN
}

A sharp Landau-Kolmogorov inequality on a finite interval is proved. The proof yields the known Landau-Kolmogorov inequality on $R$ as a limiting case, and thus provides a new proof for that result.

\section{INTRODUCTION}

In 1913, Landau [11] proved that

$$
\left\|f^{(\ell)}\right\|_{I} \leqslant C_{n, \ell}\|f\|_{I}^{1-(\ell / n)}\left\|f^{(n)}\right\|_{I}^{(\ell / n)}, \quad 1 \leqslant \ell \leqslant n-1
$$

for $n=2, I=R$ or $I=R^{+}$with the sharp constants $\sqrt{2}$ and 2 , respectively. (Here $\ell$ and $n$ are integers, and the norm is the sup norm: $\left.\|f\|_{I}=\sup _{x \in I}|f(x)|.\right)$ In 1939, Kolmogorov [10] solved (1.1) on $R$ for all $n$ and $\ell$ and determined the best constants. There are several alternate proofs of (1.1) for $I=R$ of which we mention those by Bang [1], Cavaretta [3], and de Boor and Schoenberg [2].

Hadamard [7], Gorny [6] and Matorin [12] were concerned with (1.1) for $I=R^{+}$, but their constants were not optimal when $n \geqslant 4$. In 1970, Schoenberg and Cavaretta [14] gave a procedure to find the best constant for the inequality for $I=R^{+}$, and all $n$ and $\ell$. The constants were given as limits of some sequences and are not explicit.

Several papers have dealt with inequalities similar to (1.1) on a finite interval. Of these, we mention Gorny [6], Kallioniemi [8], Pinkus [13] and Fabry [5]. In the present work, Chebyshev-Euler splines are used to prove the inequality generalising the LandauKolmogorov-Gorny inequality with the best constant in some sense. These results are generalisations of works by Fabry [5] and Kallioniemi [8]. We shall prove that

$$
\left\|f^{(\ell)}\right\|_{[-1+\delta, 1-\delta]} \leqslant \frac{\left|T_{n, k}^{(\ell)}(0)\right|}{\rho_{n, k}^{1-(\ell / n)}\left(2^{n-1} \cdot n !\right)^{\ell / n}}\|f\|_{[-1,1]}^{1-(\ell / n)}\left\|f^{(n)}\right\|_{[-1,1]}^{(\ell / n)}
$$

Received 1st February, 1993.

Research done in partial fulfillment of the Ph.D. degree at the University of Alberta under supervision of Professor Z. Ditzian. The author is indebted to Professor Z. Ditzian for his generous advice and encouragement throughout this work.

Copyright Clearance Centre, Ine. Serial-fee code: 0004-9729/93 \$A2.00+0.00. 
where $T_{n, k}(x)$ is the Chebyshev-Euler spline of degree $n$ with $k$ knots, $\rho_{n, k}=$ $\left\|T_{n, k}\right\|_{[-1,1]}$ and $\delta=\left[\left(2^{n-1} \cdot n !\|f\|_{[-1,1]}\right) /\left(\rho_{n, k}\left\|f^{(n)}\right\|_{[-1,1]}\right)\right]^{1 / n}$. The constant $\left(\left|T_{n, k}^{(\ell)}(0)\right|\right) /\left(\rho_{n, k}^{1-(\ell / n)}\left(2^{n-1} \cdot n !\right)^{\ell / n}\right)$ can not be replaced by any smaller one.

If we use a sequence of intervals $\left[-A_{\ell}, A_{\ell}\right]$ such that $A_{\ell} \rightarrow \infty$, we can derive a new proof of Kolmogorov's theorem for $R$. Therefore, one obtains a uniform approach to the Landau-Kolmogorov problem by using the Chebyshev-Euler splines (see also Schoenberg and Cavaretta [14] for $I=R^{+}$).

\section{Properties of the Chebyshev-Euler splines}

In order to solve the Landau problem on a finite interval, we consider the following perfect splines defined on the interval $I=[-1,1]$ :

$$
T(x)=2^{n-1} x^{n}+\sum_{i=1}^{k}(-1)^{i} 2^{n}\left(x-\xi_{i}\right)_{+}^{n}+\sum_{j=0}^{n-1} a_{j} x^{j}
$$

where $a_{j}, 0 \leqslant j<n$ and $\xi_{i}, 1 \leqslant i \leqslant k$ are free parameters, and

$$
-1<\xi_{1}<\xi_{2}<\cdots<\xi_{k}<1 .
$$

Let $\mathbb{T}$ be the collection of all perfect splines of the form (2.1).

Definition 2.1: We define the perfect spline $T_{n, k}(x)$ as the function of form (2.1) such that

$$
\left\|T_{n, k}\right\|_{I}=\inf _{T \in \mathbb{T}}\|T\|_{I}
$$

We call $T_{n, k}(x)$ the Chebyshev-Euler spline of degree $n$ with $k$ knots (see [4] and [14]).

If for $T(x) \in \mathbb{T}$ there are $m$ points $-1 \leqslant t_{1} \leqslant t_{2} \leqslant \ldots \leqslant t_{m} \leqslant 1$ such that

$$
T\left(t_{i}\right)=(-1)^{i_{0}+i}\|T\|_{I}, \quad 1 \leqslant i \leqslant m
$$

for some fixed $i_{0}(0$ or 1$)$, we say that $T(x)$ has $m$ points of equioscillation.

Now, we cite an important theorem from [4], yielding some basic properties of the Chebyshev-Euler splines. In the next section, we shall use these properties to prove our main results. This theorem guarantees the existence and uniqueness of $T_{n, k}(x)$.

TheOREM 2.2. (Cavaretta [4].) There is a unique perfect spline $T_{n, k}(x)$ of degree $n$ with $k$ simple knots satisfying (2.3). $T_{n, k}(x)$ has precisely $n+k+1$ points of equioscillation, and is in fact the Chebyshev-Euler spline.

The following proposition was stated in [14] but no proof was given there. For the sake of completeness, we shall prove it here. 
Proposition 2.3. For $T_{n, k}(x)$ given in Definition 2.1,

$$
T_{n, k}(-x)=(-1)^{n+k} T_{n, k}(x)
$$

Proof: Suppose $-1<\xi_{1}<\xi_{2}<\cdots<\xi_{k}<1$ are the $k$ simple knots of $T_{n, k}(x)$, and

$$
T_{n, k}(x)=2^{n-1} x^{n}+\sum_{i=1}^{k}(-1)^{i} 2^{n}\left(x-\xi_{i}\right)_{+}^{n}+\sum_{\ell=0}^{n-1} a_{\ell} x^{\ell} .
$$

Since

$$
\left(-x-\xi_{i}\right)_{+}^{n}=(-1)^{n}\left(x+\xi_{i}\right)^{n}-(-1)^{n}\left(x+\xi_{i}\right)_{+}^{n},
$$

we have

$$
\begin{aligned}
& T_{n, k}(-x)=(-1)^{n}\left[2^{n-1} x^{n}+\sum_{i=1}^{k} 2^{n}(-1)^{i} x^{n}+\sum_{i=1}^{k}(-1)^{i} \sum_{\ell=0}^{n-1}\left(\begin{array}{l}
n \\
\ell
\end{array}\right) \xi_{i}^{n-\ell} x^{\ell}\right. \\
&\left.\quad+\sum_{\ell=0}^{n-1}(-1)^{n+\ell} a_{\ell} x^{\ell}\right] \\
&=(-1)^{n+k}\left[2^{n-1} x^{n}+\sum_{j=1}^{k}(-1)^{j} 2^{n}\left(x-\eta_{j}\right)_{+}^{n}+P_{n-1}(x)\right] \\
& \equiv(-1)^{n+k} \widehat{T}_{n, k}(x)
\end{aligned}
$$

where $j=k-i+1, \xi_{i}=-\eta_{k-i+1}=-\eta_{j}$, and

$$
P_{n-1}(x)=(-1)^{k} \sum_{\ell=0}^{n-1}\left[(-1)^{n+\ell} a_{\ell}+2^{n}\left(\begin{array}{l}
n \\
\ell
\end{array}\right) \sum_{i=1}^{k}(-1)^{i} \xi_{i}^{n-\ell}\right] x^{\ell}
$$

is a polynomial of degree $n-1$. Thus $\widehat{T}_{n, k}(x)$ is a perfect spline of the form (2.1), and $\left\|T_{n, k}\right\|_{I}=\left\|\widehat{T}_{n, k}\right\|_{I}$. Therefore, by the uniqueness of $T_{n, k}(x)$, we have

and

$$
T_{n, k}(x)=\widehat{T}_{n, k}(x),
$$

$$
\xi_{i}=-\xi_{k-i+1}, \quad i=1,2, \ldots, k \text {. }
$$

This completes the proof of Proposition 2.3.

Proposition 2.4: (Karlin [9]). Suppose $\rho_{n, k} \equiv\left\|T_{n, k}\right\|_{I}$ with $T_{n, k}(x)$ satisfying (2.3). Then $\rho_{n, k}$ is strictly decreasing in $k$ and

$$
\lim _{k \rightarrow+\infty} \rho_{n, k}=0
$$

$[9$, p.409, Lemma 5.7.] 


\section{The Main Results}

In this section we discuss the main results of the paper. First we prove (1.2) and give another version of the Landau-Kolmogorov inequality on the finite interval. Then we derive a new proof of Kolmogorov's theorem on the real line $R$.

In order to prove (1.2), we need the following key result, which was proved in [8] for $k=0$. In that case, $T_{n, k}(x)$ is exactly the Chebyshev polynomial of degree $n$.

THEOREM 3.1. Let $f(x) \in C^{n-1}[-1,1]$ and $f^{(n-1)}(x)$ be absolutely continuous such that

$$
\|f\| \leqslant \rho_{n, k}, \quad\left\|f^{(n)}\right\| \leqslant 2^{n-1} \cdot n ! .
$$

Then, for even $n+k+\ell$ and $1 \leqslant \ell \leqslant n-1$, we have

$$
\left|f^{(\ell)}(0)\right| \leqslant\left|T_{n, k}^{(\ell)}(0)\right|
$$

The constant $\left|T_{n, k}^{(\ell)}(0)\right|$ on the right hand side of (3.1) cannot be replaced by any smaller one.

Proof: Without loss of generality, we assume that $n+k$ and $\ell$ are both odd. (The case where both $n+k$ and $\ell$ are even can be treated in a similar manner.) Set

$$
F(x)=(f(x)-f(-x)) / 2 .
$$

Then $F(x)$ and $T_{n, k}(x)$ are both odd functions, and

$$
F^{(i)}(x)=\left(f^{(i)}(x)-(-1)^{i} f^{(i)}(-x)\right) / 2, \quad 0 \leqslant i \leqslant n .
$$

Hence

and

$$
\left|F^{(\ell)}(0)\right|=\left|f^{(\ell)}(0)\right|
$$

$$
\|F\| \leqslant \rho_{n, k}, \quad\left\|F^{(n)}\right\| \leqslant 2^{n-1} \cdot n ! .
$$

We now have only to show that

$$
\left|F^{(\ell)}(0)\right| \leqslant\left|T_{n, k}^{(\ell)}(0)\right| .
$$

Assuming this is not so, there exists a constant $\alpha, \alpha>1$, or $\alpha<-1$, such that

$$
F^{(\ell)}(0)=\alpha T_{n, k}^{(\ell)}(0)
$$

We assume $\alpha>1$ and the case $\alpha<-1$ can be treated in a similar manner. Define $h(x):[-1,1] \rightarrow R$ by

$$
h(x) \equiv \alpha T_{n, k}(x)-F(x),
$$


then $h(x)$ is an odd function.

Since $\|F\| \leqslant \rho_{n, k}$ and $T_{n, k}(x)$ has $n+k+1$ points of equioscillation by Theorem 2.2, $h(x)$ must have at least $n+k$ zeros in $[-1,1]$. By Rolle's theorem, $h^{(\ell-1)}(x)$ must then have at least $n+k+1-\ell$ zeros in $(-1,1)$. Observing also that $h^{(\ell-1)}(x)$ is an odd function, $h^{(\ell-1)}(0)=0$. Thus, by Rolle's theorem again, $h^{(\ell)}(x)$ must have at least $n+k-\ell$ zeros in $(-1,0) \cup(0,1)$. On the other hand, by the definition of $h(x), h^{(\ell)}(0)=0$. Therefore, $h^{(\ell)}(x)$ has at least $n+k-\ell+1$ zeros in $(-1,1)$ and $h^{(n-1)}(x)$ will have at least $k+2$ zeros in $(-1,1)$. This implies that there exists an integer $i_{0}, 1 \leqslant i_{0} \leqslant k-1$, such that $h^{(n-1)}(x)$ has at least two zeros in $\left[\xi_{i_{0}}, \xi_{i_{0}+1}\right]$. We select two of these zeros, say $\eta_{1}$ and $\eta_{2}$, and assume $\eta_{1}<\eta_{2}$. Thus,

$$
\begin{aligned}
0=\left|h^{(n-1)}\left(\eta_{2}\right)\right| & =\left|h^{(n-1)}\left(\eta_{2}\right)-h^{(n-1)}\left(\eta_{1}\right)\right| \\
& =\left|\int_{\eta_{1}}^{\eta_{2}}\left(\alpha T_{n, k}^{(n)}(x)-F^{(n)}(x)\right) d x\right| \\
& \geqslant \alpha\left(\eta_{2}-\eta_{1}\right) 2^{n-1} \cdot n !-\left(\eta_{2}-\eta_{1}\right) 2^{n-1} \cdot n !>0,
\end{aligned}
$$

which is a contradiction. If we let $f(x)$ be $T_{n, k}(x)$, then (3.1) becomes an equality.

ThEOREM 3.2. Let $f(x) \in C^{n-1}[-1,1]$ and $f^{(n-1)}(x)$ be absolutely continuous, then for an even integer $n+k+\ell$,

$$
\left\|f^{(\ell)}\right\|_{[-1+\delta, 1-\delta]} \leqslant \frac{\left|T_{n, k}^{(\ell)}(0)\right|}{\rho_{n, k}^{1-(\ell / n)}\left(2^{n-1} \cdot n !\right)^{\ell / n}}\|f\|^{1-(\ell / n)}\left\|f^{(n)}\right\|^{\ell / n}
$$

where $\delta=\left(\left(2^{n-1} \cdot n !\|f\|\right) /\left(\rho_{n, k}\left\|f^{(n)}\right\|\right)\right)^{1 / n}$ and $1 \leqslant \ell \leqslant n-1$. Furthermore, the constant on the right hand side of (3.2) cannot be replaced by any smaller one.

Proof: For any $x_{0} \in[-1+\delta, 1-\delta]$, define $F(x):[-1,1] \rightarrow R$ by

$$
F(x)=\rho_{n, k} f\left(x_{0}+\delta x\right) /\|f\| .
$$

Then

$$
\|F\| \leqslant \rho_{n, k}, \quad\left\|F^{(n)}\right\| \leqslant 2^{n-1} \cdot n !,
$$

and

$$
\left|F^{(\ell)}(x)\right|=\rho_{n, k} \delta^{\ell} f^{(\ell)}\left(x_{0}+\delta x\right) /\|f\| \text {. }
$$

Applying Theorem 3.1, we have

$$
\begin{aligned}
\left|f^{(\ell)}\left(x_{0}\right)\right| & =\left|F^{(\ell)}(0)\right|\|f\| /\left(\rho_{n, k} \cdot \delta^{\ell}\right) \\
& \leqslant \frac{\left|T_{n, k}^{(\ell)}(0)\right|}{\rho_{n, k}^{1-(\ell / n)}\left(2^{n-1} \cdot n !\right)^{\ell / n}}\|f\|^{1-(\ell / n)}\left\|f^{(n)}\right\|^{\ell / n} .
\end{aligned}
$$

If we let $f(x)$ be $T_{n, k}(x)$, then $\delta=1$ and we have equality in (3.2). This completes the proof.

For the general finite interval $[a, b]$, using a linear transformation, we have 
Corollary 3.3. Let $f(x) \in C^{(n-1)}[a, b]$ and $f^{(n-1)}(x)$ be absolutely continuous, then for even $n+k+\ell$,

$$
\left\|f^{(\ell)}\right\|_{[a+\delta, b-\delta]} \leqslant \frac{\left|T_{n, k}^{(\ell)}(0)\right|}{\rho_{n, k}^{1-(\ell / n)}\left(2^{n-1} \cdot n !\right)^{\ell / n}}\|f\|_{[a, b]}^{1-(\ell / n)}\left\|f^{(n)}\right\|_{[a, b]}^{\ell / n}
$$

where $\delta=\left(2^{n-1} \cdot n !\|f\|_{[a, b]}\right) /\left(\rho_{n, k}\left\|f^{(n)}\right\|_{[a, b]}\right)^{1 / n}$ and $1 \leqslant \ell \leqslant n-1$.

In Theorem 3.1 we use $\left|T_{n, k}^{(\ell)}(0)\right|$ to estimate $\left|f^{(\ell)}(0)\right|$. Actually, using the same argument, we can estimate $\left|f^{(\ell)}( \pm 1)\right|$ by $\left|T_{n, k}^{(\ell)}( \pm 1)\right|$. This is a generalisation of Theorem 1 in [5] (that theorem was proved only for Chebyshev polynomials).

THEOREM 3.4. Suppose $f(x)$ satisfies the conditions in Theorem 3.1. Then, for $1 \leqslant \ell \leqslant n-1$, we have

$$
\left|f^{(\ell)}( \pm 1)\right| \leqslant\left|T_{n, k}^{(\ell)}( \pm 1)\right|
$$

The constant $\left|T_{n, k}^{(\ell)}( \pm 1)\right|$ cannot be replaced by any smaller one.

REMARK. A stronger result than Theorem 3.4 was obtained by Schoenberg and Cavaretta in [14]. In fact, the interval can be a little smaller, but the proof there is quite complicated and only a sketch of the proof is given.

Using Theorem 3.4, we can also estimate the two parts of the interval $[-1,1]$ adjacent to \pm 1 . Thus, combining with Theorem 3.1, we shall obtain another version of the Landau-Kolmogorov inequality on the finite interval. This improves the result of Theorem 2 in [5], in particular, for the middle part of the interval.

TheOREM 3.5. Let $f(x) \in C^{n-1}[-1,1]$ and $f^{(n-1)}(x)$ be absolutely continuous, then for $n+k+\ell$ even and $1 \leqslant \ell \leqslant n-1$,

$$
\left\|f^{(\ell)}\right\|_{I_{i}} \leqslant\left|T_{n, k}^{(\ell)}(i)\right|\left(\frac{\|f\|}{\rho_{n, k}}\right)^{1-(\ell / n)}\left[\max \left\{\frac{\left\|f^{(n)}\right\|}{2^{n-1} \cdot n !},\left(\frac{3}{2}\right)^{n} \frac{\|f\|}{\rho_{n, k}}\right\}\right]^{\ell / n}
$$

where $I_{i}=[-1+2(i+1) / 3,-1+2(i+2) / 3], i=-1,0,1$.

Proof: For $i=-1,0,1$, let $x_{0} \in I_{i}$ and define $F_{i}(x):[-1,1] \rightarrow R$ by

$$
F_{i}(x)=\rho_{n, k} f\left(x_{0}+(x-i) \mu\right) /\|f\|
$$

where $\mu=\min \left\{2 / 3,\left[2^{n-1} \cdot n !\|f\| /\left(\rho_{n, k}\left\|f^{(n)}\right\|\right)\right]^{1 / n}\right\}$. Then, $F_{i}(x)$ is well defined, and

$$
\left\|F_{i}\right\| \leqslant \rho_{n, k}, \quad\left\|F_{i}^{(n)}\right\| \leqslant 2^{n-1} \cdot n !, \quad i=-1,0,1 .
$$


Applying Theorem 3.4 or Theorem 3.1 and observing that

$$
\left|f^{(\ell)}\left(x_{0}\right)\right|=\|f\|\left|F^{(\ell)}(i)\right| /\left(\rho_{n, k} \mu^{\ell}\right), \quad i=-1,0,1,
$$

we have

$$
\left|f^{(\ell)}\left(x_{0}\right)\right| \leqslant\left|T_{n, k}^{(\ell)}(i)\right|\left(\frac{\|f\|}{\rho_{n, k}}\right)^{1-(\ell / n)}\left[\max \left\{\frac{\left\|f^{(n)}\right\|}{2^{n-1} \cdot n !},\left(\frac{3}{2}\right)^{n} \frac{\|f\|}{\rho_{n, k}}\right\}\right]^{\ell / n} .
$$

This completes the proof of Theorem 3.5 .

REMARK. Since $n+k+\ell$ can be any integer (even or odd) in Theorem 3.4, $n+k+\ell$ can be odd in the inequality (3.5) for $i= \pm 1$. It is also unnecessary to divide $[-1,1]$ into three equal parts, but in this case, the constant $(3 / 2)^{n}$ in front of $\|f\| / \rho_{n, k}$ will be replaced by a different constant.

In Corollary 3.3, one can obtain the inequality (3.5) by a linear transformation for a general finite interval $[a, b]$. Now we can derive a new proof of the Landau-Kolmogorov inequality on $R$.

For convenience, we normalise $T_{n, k}(x)$ first, writing

$$
S_{n, k}(x)=\rho_{n, k}^{-1} T_{n, k}\left(\rho_{n, k}^{1 / n} x\right) .
$$

Clearly $S_{n, k}(x)$ is defined on $\left[-\rho_{n, k}^{-(1 / n)}, \rho_{n, k}^{-(1 / n)}\right]$, and satisfies

$$
\left\|S_{n, k}\right\|=1, \quad\left\|S_{n, k}^{(n)}\right\|=2^{n-1} \cdot n !
$$

LEMMA 3.6. For $S_{n, k}(x)$ defined in (3.6), we have

$$
\left|S_{n, 0+i}^{(\ell)}(0)\right| \geqslant\left|S_{n, 2+i}^{(\ell)}(0)\right| \geqslant \ldots \geqslant\left|S_{n, 2 k+i}^{(\ell)}(0)\right| \geqslant \cdots, \quad i=0 \text { or } 1
$$

where $1 \leqslant \ell \leqslant n-1$ and $n+\ell+i$ is even.

Proof: Without loss of generality, assume that $i=0$ and $n+\ell$ is even. Set

$$
F_{n, 2 k+2}(x)=\frac{\rho_{n, 2 k}}{\rho_{n, 2 k+2}} T_{n, 2 k+2}\left(\left(\frac{\rho_{n, 2 k+2}}{\rho_{n, 2 k}}\right)^{1 / n} x\right) \text {. }
$$

Since $\rho_{n, 2 k+2} / \rho_{n, 2 k} \leqslant 1, F_{n, 2 k+2}(x)$ is well defined on $[-1,1]$, and

$$
\left\|F_{n, 2 k+2}\right\| \leqslant \rho_{n, 2 k}, \quad\left\|F_{n, 2 k+2}^{(n)}\right\| \leqslant 2^{n-1} \cdot n ! .
$$

By Theorem 3.1, 


$$
\left|F_{n, 2 k+2}^{(\ell)}(0)\right|=\frac{\rho_{n, 2 k}}{\rho_{n, 2 k+2}}\left(\frac{\rho_{n, 2 k+2}}{\rho_{n, 2 k}}\right)^{\ell / n}\left|T_{n, 2 k+2}^{(\ell)}(0)\right| \leqslant\left|T_{n, 2 k}^{(\ell)}(0)\right|
$$

or

$$
\frac{\left|T_{n, 2 k}^{(\ell)}(0)\right|}{\rho_{n, 2 k}^{1-(\ell / n)}} \geqslant \frac{\left|T_{n, 2 k+2}^{(\ell)}(0)\right|}{\rho_{n, 2 k+2}^{1-(\ell / n)}}
$$

Thus

$$
\left|S_{n, 2 k}^{(\ell)}(0)\right| \geqslant\left|S_{n, 2 k+2}^{(\ell)}(0)\right|
$$

THEOREM 3.7. Let $f(x) \in C^{n-1}(-\infty, \infty)$ and $f^{(n-1)}(x)$ be absolutely continuous, then

$$
\left\|f^{(\ell)}\right\|_{(-\infty, \infty)} \leqslant C_{n, \ell}\|f\|_{(-\infty, \infty)}^{1-(\ell / n)}\left\|f^{(n)}\right\|_{(-\infty, \infty)}^{\ell / n}
$$

where $C_{n, \ell}=\lim _{k \rightarrow \infty}\left|S_{n, 2 k+i}^{(\ell)}(0)\right| /\left(2^{n-1} \cdot n !\right)^{\ell / n}$, and $i=0$ or 1 such that $n+\ell+i$ is even. Moreover, $C_{n, \ell}$ is Kolmogorov's constant for $R$.

Proof: Suppose that $i=0$ and $n+\ell$ is even. Applying Corollary 3.3, we have

$$
\left\|f^{(\ell)}\right\|_{(-\infty, \infty)} \leqslant \frac{\left|S_{n, 2 k}^{(\ell)}(0)\right|}{\left(2^{n-1} \cdot n !\right)^{\ell / n}}\|f\|_{(-\infty, \infty)}^{1-(\ell / n)}\left\|f^{(n)}\right\|_{(-\infty, \infty)}^{\ell / n}
$$

Since $k$ is arbitrary, and by Lemma 3.6,

$$
\left\|f^{(\ell)}\right\|_{(-\infty, \infty)} \leqslant C_{n, \ell}\|f\|_{(-\infty, \infty)}^{1-(\ell / n)}\left\|f^{(n)}\right\|_{(-\infty, \infty)}^{\ell / n} .
$$

Now, consider the function sequence $\left\{S_{n, 2 k}(x)\right\}_{k=0}^{\infty}$. Let $N$ be any integer. By Proposition 2.4, there exists an integer $K$ such that

$$
\rho_{n, 2 k}^{-(1 / n)} \geqslant N+1, \quad \text { for } k \geqslant K .
$$

Using the definition of $S_{n, 2 k}(x)$ and applying Theorem 3.4, we now have

$$
\left\|S_{n, 2 k}^{(\ell)}\right\|_{[-N, N]} \leqslant\left|T_{n, 0}^{(\ell)}( \pm 1)\right|, \quad 0 \leqslant \ell \leqslant n, k \geqslant K .
$$

Hence, for any $x_{1}, x_{2} \in[-N, N]$, we have

$$
\left|S_{n, 2 k}^{(\ell)}\left(x_{1}\right)-S_{n, 2 k}^{(\ell)}\left(x_{2}\right)\right| \leqslant\left|T_{n, 0}^{(\ell+1)}( \pm 1)\right|\left|x_{1}-x_{2}\right|, \quad 0 \leqslant \ell \leqslant n-1, k \geqslant K .
$$


Therefore the functions $\left\{S_{n, 2 k}^{(\ell)}(x)\right\}_{k=0}^{\infty}(0 \leqslant \ell \leqslant n-1)$ are uniformly bounded and equicontinuous on $[-N, N]$.

Using the Arzela-Ascoli theorem, we can find a subsequence $\left\{S_{n, 2 k_{i}}(x)\right\}_{i=1}^{\infty}$ of $\left\{S_{n, 2 k}(x)\right\}_{k=K}^{\infty}$, such that $\left\{S_{n, 2 k_{i}}^{(\ell)}(x)\right\}_{i=1}^{\infty}(0 \leqslant \ell \leqslant n-1)$ are all uniformly convergent on $[-N, N]$. By the diagonalisation process, we pick a subsequence $\left\{S_{n, 2 k_{j}}(x)\right\}_{j=1}^{\infty}$ of $\left\{S_{n, 2 k_{i}}(x)\right\}_{i=1}^{\infty}$, such that $\left\{S_{n, 2 k_{j}}^{(\ell)}(x)\right\}_{j=1}^{\infty}(0 \leqslant \ell \leqslant n-1)$ are all uniformly convergent on any finite interval.

The limit function of the above process, $E_{n}(x)$, satisfies $E_{n}(x) \in C^{n-1}(-\infty, \infty)$, $E_{n}^{(n-1)}(x)$ is absolutely continuous,

and

$$
\left\|E_{n}\right\|_{(-\infty, \infty)} \leqslant 1, \quad\left\|E_{n}^{(n)}\right\|_{(-\infty, \infty)} \leqslant 2^{n-1} \cdot n !,
$$

$$
\left|E_{n}^{(\ell)}(0)\right|=\lim _{k \rightarrow \infty}\left|S_{n, 2 k}^{(\ell)}(0)\right|, \quad 0 \leqslant \ell \leqslant n-1 .
$$

Therefore, $E_{n}(x)$ is an extremal function of (3.8), and $C_{n, \ell}$ should be Kolmogorov's constant for $R$. This completes the proof.

By Kolmogorov's theorem, we know $C_{n, \ell}$ explicitly, but it is difficult to calculate $S_{n, 2 k+i}^{(\ell)}(0)$ for large $n$ and $k$. However, Theorem 3.7 established the relation between Kolmogorov's constant $C_{n, \ell}$ and $\left\{S_{n, 2 k+i}^{(\ell)}(0)\right\}_{k=0}^{\infty}$. For $n=2$ or 3 , we can calculate $S_{n, 2 k+i}^{(\ell)}$, which yields exactly Kolmogorov's constants $C_{n, \ell}$. Actually all terms in (3.7) have the same value for $n=2$ and $n=3$.

\section{REFERENCES}

[1] T. Bang, 'Une inégalité de Kolmogorof et les fonctions presque-periodiques', Danske Vid. Selsk. Math. Fos. Medd. 19 (1941).

[2] C. de Boor and I.J. Schoenberg, 'Cardinal interpolation and spline functions VIII. The Budan-Fourier theorem for splines and applications', in Spline functions Karlsrude 1975, (K. Böhmer, G. Meinardus and W. Schempp, Editors), Lecture Notes in Mathematics 501 (Springer-Verlag, Berlin, Heidelberg, New York, 1976), pp. 1-79.

[3] A.S. Caravetta, 'An elementary proof of Kolmogorov's theorem', Amer. Math. Monthly 81 (1974), 480-486.

[4] A.S. Caravetta, 'Oscillatory and zero properties for perfect splines and monosplines', $J$. Analyse Math. 28 (1975), 41-59.

[5] C. Fabry, 'An elementary proof of Gorny's inequality', Proc. Royal Soc. Edinburgh Sect. A 105 (1987), 345-349.

[6] A. Gorny, 'Contribution à l'étude des fonctions dérivables d'une variable réelle', Acta Math. 71 (1939), 317-358.

[7] J. Hadamand, 'Sur le module maximum d'une fonction et de ses dérivées', C.R. Soc. Math. France (1914), 68-72. 
[8] H. Kallioniemi, 'On bounds for the derivatives of a complex-valued function on a compact interval', Math. Scand. 39 (1976), 295-314.

[9] S. Karlin, 'Oscillatory perfect splines and related extremum problems', in Spline functions and approximation theory, (S. Karlin, C. A. Micchelli, A. Pinkus and I. J. Schoenberg, Editors) (Academic Press, New York, 1976), pp. 371-460.

[10] A. Kolmogorov, 'On inequalities between the upper bounds of the successive derivatives of an arbitrary function on an infinite interval', Amer. Math. Soc. Transl. Ser. 12 (1962), 233-243.

[11] E. Landau, 'Einige Ungleichungen für zweimal differentzierbare Funktionen', Proc. London Math. Soc. (2) 13 (1913), 43-49.

[12] A.P. Matorin, 'On inequalities between the maxima of the absolute values of a function and its derivatives on a half-line', Amer. Math. Soc. Transl. Ser. 28 (1958), 13-17.

[13] A. Pinkus, 'Some extremal properties of perfect splines and the pointwise Landau problem on the finite interval', J. Approx. Theory 23 (1978), 37-64.

[14] I.J. Schoenberg and A. Cavaretta, Solution of Landau's problem concerning higher derivatives on the halfine (MRCT.S.R. \#1050, Madison, Wisconsin, 1970).

Department of Mathematics

Statistics \& Computing Science

Dalhousie University

Halifax, Nova Scotia

Canada B3H 3J5 